Victoria. Compared with elsewhere in Australia, Victorians were less likely to report sex in the last 4 weeks $(54.8 \%$ vs $68.0 \%$, diff $=-13.3 \%, 95 \%$ CI $-19.6,-6.9)$. Victorians were also less likely to report casual hook-ups $(10.6 \%$ vs $17.3 \%$; diff $=-6.7 \%, 95 \% \mathrm{CI}-12.5,-0.8)$ and sex with a fuckbuddy $(10.6 \%$ vs $16.9 \%$; diff $=-6.2 \%$, 95\%CI-12.1,$0.4)$. There was no difference in dating app use in the last 4 weeks between Victorians and others $(27.0 \%$ vs $26.6 \%$; diff $=0.4 ; 95 \% \mathrm{CI}-5.2,6.1)$, but Victorians were more likely to report using apps for virtual dates $(15.7 \%$ vs $2.9 \%$, diff $=12.8 \%$, 95\% CI $6.2,19.3)$ and chatting $(83.0 \%$ vs $72.5 \%$; diff $=10.5 ; 95 \% \mathrm{CI} 0.0,20.9)$ and less likely to use them for face-to-face dates $(15.1 \%$ vs $56.9 \%$; diff $=-41.8 \%$, 95\% CI $-52.9,-30.7)$.

Conclusions Hard lockdowns impact sexual behaviour and practices. However, sexual activity did not stop completely, highlighting the importance of ensuring accessibility of sexual and reproductive health services during periods of restriction.

\section{P094 MOTIVATIONAL INTERVIEWING AND THE PREVENTION OF STIS DURING PREGNANCY: COMING UP 'EMPTY'}

${ }^{1} \mathrm{~S}$ Williams*, ${ }^{1} \mathrm{P}$ Loosier, ${ }^{1,2} \mathrm{~A}$ Machefsky. ${ }^{1}$ Centers for Disease Control and Prevention, Atlanta, USA; ${ }^{2}$ Gilstrap Fellowship, Atlanta, USA

\subsection{6/sextrans-2021-sti.223}

Background Sexually transmitted infections (STIs) risk persist during pregnancy. Of special concern, rates of congenital syphilis continue to rise in the US and elsewhere. One effective approach for helping pregnant people reduce diverse maternal and neonatal risks is motivational interviewing (MI), which has demonstrated effectiveness in reducing alcohol/substance misuse and smoking during pregnancy, and STI risk among non-pregnant youth and adults. We sought to describe MI's role in STI prevention in pregnancy to help inform the field of best practices.

Methods We attempted a systematic review of the use of MI for STI prevention in pregnant women in PubMed, MEDLINE, Ovid, Embase and Scopus databases. Articles included for consideration were peer-reviewed studies conducted in the US, published between 2000 and 2020, in English, included women who were pregnant at the onset of the study, and reported STI outcomes. Articles were excluded if women in the sample became pregnant as a result of intervention failure. Results Of 595 citations, only one article was eligible for fulltext review; all others failed to meet the inclusion criteria. This yielded an 'empty' review, despite substantial literature and MI's noted efficacy.

Conclusion Arguments to support MI's use to prevent STIs or re-infection among pregnant people are based on its success in this population in addressing other behavioral risks (alcohol and drug use). Despite its promise, published research in this area is absent. MI is proven to be effective, versatile, and has demonstrated success in promoting a multitude of health-benefiting and risk reducing behavioral changes among pregnant people. Our 'empty review' underscores the need for attention in this area. Future research could compare interventions integrating MI to the standard of care to measure the effect on STI prevention among pregnant people.

\section{P095 GENERAL PRACTITIONER VIEWS TOWARDS PATIENT DELIVERED PARTNER THERAPY FOR CHLAMYDIA INFECTION IN AUSTRALIA}

1) Goller*, 'I Coombe, ${ }^{1} \mathrm{H}$ Bittleston, ${ }^{2} \mathrm{C}$ Bourne, ${ }^{3} \mathrm{D}$ Bateson, ${ }^{1} \mathrm{~A}$ Vaisey, ${ }^{4} \mathrm{~J}$ Tomnay, ${ }^{5} \mathrm{O}$ Donnell $\mathrm{H},{ }^{6} \mathrm{C}$ Garrett, ${ }^{7} \mathrm{C}$ Estcourt, ${ }^{8} \mathrm{M}$ Temple-Smith, ${ }^{1} \mathrm{~J}$ Hocking. ${ }^{1}$ The University of Melbourne; School of Population and Global Health, Carlton, Australia; ${ }^{2}$ STI Programs Unit, NSW Ministry of Health, Sydney, Australia; ${ }^{3}$ Family Planning NSW, Ashfield, Australia; ${ }^{4}$ The University of Melbourne, Centre for Excellence in Rural Sexual Health, Shepparton, Australia; ${ }^{5}$ Victorian Government, Department of Health and Human Services, Melbourne, Australia; ${ }^{6}$ Family Planning Victoria, Box Hill, Australia; ${ }^{7}$ Glasgow Caledonian University, School of Health and Life Science, Glasgow, UK; ${ }^{8}$ The University of Melbourne, Department of General Practice, Melbourne, Australia

\subsection{6/sextrans-2021-sti.224}

Background Patient delivered partner therapy (PDPT) refers to the process in which antibiotic treatment for chlamydia infection is prescribed or provided to an index case to pass onto their sexual partner/s. Since 2015, health authority guidance for PDPT has been provided in some areas of Australia. However evidence regarding PDPT use in Australia are limited. We investigated recent use and perceptions of PDPT for chlamydia among general practitioners (GPs) working in Australia.

Methods During 2019 we conducted an online survey comprising multiple-choice and open-ended questions to investigate GPs' chlamydia management practices, including PDPT. We conducted logistic regression to identify factors associated with offering PDPT and directed content analysis of free-text data to explore GPs' perceptions towards PDPT.

Results A total of 323 GPs responded to the survey, $85.8 \%$ $(\mathrm{n}=277)$ answered PDPT-focused questions, providing 628 free-text comments. Over half (53.4\%) reported never offering PDPT while $36.5 \%$ sometimes and 10.1\% often offered PDPT. GPs more likely to offer PDPT were aged $\geq 55$ years (adjusted odds ratio, AOR 2.8, 95\%CI 1.4-5.7), worked in non-metropolitan areas (AOR 2.5, 95\%CI 1.5-4.4) and in Australian States/Territories with health authority PDPT guidance (AOR 2.3, 95\%CI 1.4-3.9). Qualitative data showed many GPs recognised PDPT's potential to treat difficult to engage partners but expressed hesitancy to offer PDPT because they considered it best practice for partners to attend care. A case-by-case approach that considered patient and partner circumstances to determine PDPT suitability was emphasised. Many GPs indicated a need for professional and health authority guidance that PDPT is permissible and practical resources to support its use.

Conclusions GPs appear to accept the place of PDPT as targeted to those who may otherwise not access testing or treatment Availability of health authority guidance appears to have supported some GPs to incorporate PDPT into their practice.

\section{P096 INCIDENCE AND RISK FACTORS OF C. TRACHOMATIS AND N. GONORRHOEAE AMONG YOUNG WOMEN FROM THE WESTERN CAPE, SOUTH AFRICA}

${ }^{1} \mathrm{~V}$ Jongen*, ${ }^{1,2} \mathrm{M}$ Schim van der Loeff, ${ }^{3} \mathrm{M}$ Botha, ${ }^{4} \mathrm{~S}$ Sudenga, ${ }^{5} \mathrm{M}$ Abrahamsen, ${ }^{5}$ A Giuliano. 'Department of Infectious Diseases, Public Health Service Amsterdam, Amsterdam, The Netherlands; ${ }^{2}$ Amsterdam UMC, Univ of Amsterdam, Internal Medicine, Amsterdam Infection and Immunity (AlI), Amsterdam, The Netherlands; ${ }^{3}$ Department of Obstetrics and Gynaecology, Stellenbosch University, Cape Town, South Africa; ${ }^{4}$ Division of Epidemiology, Vanderbilt University Medical Center, Nashville, USA; ${ }^{5}$ Center for Immunization and Infection Research in Cancer, Moffitt Cancer Center, Tampa, United Stated of America

10.1136/sextrans-2021-sti.225 